\title{
Renal Allograft Thrombosis in the Early Post Transplant Period
}

\author{
M. Budruddin ${ }^{1 *}$, Issa Al Salmi ${ }^{2}$, R. Shilpa ${ }^{2}$ \\ ${ }^{1}$ Nephrology Department, Tawam Hospital, Al Ain, UAE \\ ${ }^{2}$ Nephrology Department, Royal Hospital, Muscat, Oman \\ Email: *drbudruddin@gmail.com, isa.al.salmi@gmail.com, ramaiah_shilpa@yahoo.co.in
}

Received June 26, 2013; revised July 23, 2013; accepted August 10, 2013

Copyright (c) 2013 M. Budruddin et al. This is an open access article distributed under the Creative Commons Attribution License, which permits unrestricted use, distribution, and reproduction in any medium, provided the original work is properly cited.

\begin{abstract}
Renal allograft thrombosis involving either the renal artery or the veins is a well known entity in clinical practice. This complication of the renal transplant surgery is more common in the early part of the post transplant period and it is usually associated with acute antibody mediated rejection. This more commonly occurs in the paediatric renal transplant and also seems to have some relation to the duration of peritoneal dialysis pretransplant. However, the occurrence of graft thrombosis in isolation without clinical or histological graft rejection is not rare. We encountered a patient in whom the renal allograft thrombosis occurred after 6 weeks of commercial renal transplantation without any histological evidence of rejection.
\end{abstract}

Keywords: Allograft Thrombosis; Immunosuppression; Cyclosporine; OKT3; Hemodialysis; Renogram; Rejection and Pyelonephritis

\section{Introduction}

Renal allograft thrombosis is not an uncommon complication of renal transplantation. It usually occurs in the early post renal transplant period. Several factors have been implicated with this notorious complication. Thrombosis can even occur in the late part of the post transplant period. Irrespective of the time of development of thrombosis the outcome is equally bad.

\section{Case Summary}

A 58-year-old Omani male with background of long standing type I diabetes along with its complications of diabetic retinopathy, Nephropathy, Neuropathy, Ischemic heart disease and paroxysmal atrial fibrillation. He was initiated on haemodialysis in late 2009. He received commercial renal transplantation in October 2010 from Pakistan and was put on triple immunosuppression with Cyclosporin, Mycophenolate and prednisolone. Details of induction immunosuppression were not available in the discharge notes.

On the $8^{\text {th }}$ post operative day on his arrival in our hospital he had mild leucocytosis, surgical wound infection, uncontrolled blood sugars and the renal functions were

\footnotetext{
${ }^{*}$ Corresponding author.
}

deranged with e-GFR $21 \mathrm{ml} / \mathrm{min}$. He was started empirically on Tazocbactam after sending the samples for cultures and blood sugars were controlled with insulin. At this stage graft kidney biopsy was proposed but patient and his relatives did not agree. An impression of delayed graft function was made and he was treated for infection. Ultrasound of the graft kidney showed good blood flow in the graft kidney and the resistive index was $0.6-0.8$ and there were no collection around the renal allograft. The renal functions gradually improved with decrease in the serum creatinine from $255 \mathrm{umol} / \mathrm{l}$ on presentation to $190 \mathrm{umol} / \mathrm{l}$ on discharge. DTPA renogram was planned but we could not get it done as the family wanted to take him to the peripheral Nephrology Unit close to their home, for further care.

During his stay there in hospital after a week he developed tenderness over the graft and the Doppler ultrasound of the graft showed perinephric collection of $5.7 \times$ $2.9 \times 1.4 \mathrm{~cm}$ with high suspicion of bleed (Figure 1). In the mean time he developed Symptomatic atrial fibrillation. Apart from the rate control drugs he was also warfarinised on the suggestion of cardiologist. Subsequent three days were uneventfull with the serum creatinine improving further to $119 \mathrm{umol} / \mathrm{l}$ and also with improvement in the graft tenderness. He was hence discharged with follow up appointment in the cardiology and Trans- 


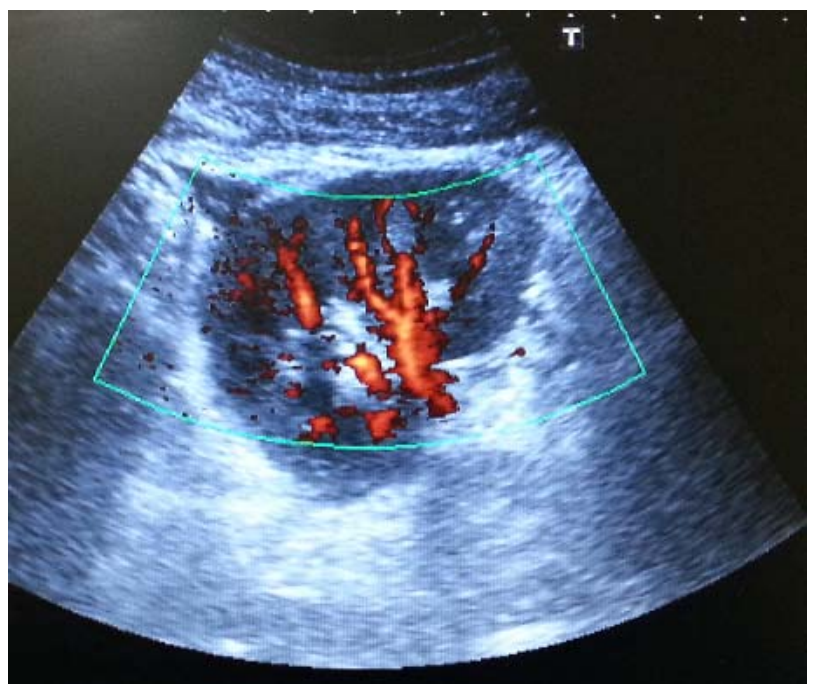

Figure 1. Perigraft haematoma shown by colour Doppler ultrasound.

plant clinic. There his medications on discharge included Ciclosporin $150 \mathrm{mg}$ bid, MMF 1gm bid, prednisolone 20 mg bid, warfarin $2 \mathrm{mg}$ od, amlodepine $10 \mathrm{mg}$ od and isosorbid $10 \mathrm{mg}$ tid.

Five days later he again presented to the emergency of that hospital with history of fever associated with chills, decreased urine output and loss of consciousness. Examination revealed hypotension blood pressure 80/50 mmhg, congested chest, bluish discolouration associated with tenderness over the right iliac fossa. Immediate resuscitation was done and the laboratory investigations showed serum creatinine of $230 \mathrm{umol} / \mathrm{l}$, Hb of $7.6 \mathrm{gm} \%$ which was earlier $9.4 \mathrm{gm} \%$ on discharge, leucocytosis of 14000 cumm and INR of 3. Urgent ultrasound of the abdomen showed increase in the size of Haematoma to 11.7 $\times 7 \times 9.1 \mathrm{~cm}$ (Figure 2). He was admitted under surgical team and started on antibiotics, fresh frozen plasma along with one dose of Vit K. Sequential ultrasound assesment of the collection was planned and the second ultrasound done the following day alarmingly showed no parenchymal blood flow and the renal functions worsened with exponential rise in serum creatinine to $>800 \mathrm{umol} / \mathrm{l}$ and anuria. He was started on haemodialysis and CT scan of the abdomen was done there showed large perinephric collection with necrotic renal allograft and air bubbles suggestive of emphysematous pyelonephritis. He was shifted to our centre on $31^{\text {st }}$ October for graft Nephrectomy. On admission in our centre he was septic with abdominal distention and severe tenderness over the Right side of the abdomen. There was leucocytosis, thrombocytopenia and the coagulation was deranged with fibrinogen degradation products (FDP) elevated. There was no evidence of Deep vein thrombosis of the lower limbs by Doppler ultrasound. He was urgently taken for exploration which showed pale looking necrotic graft

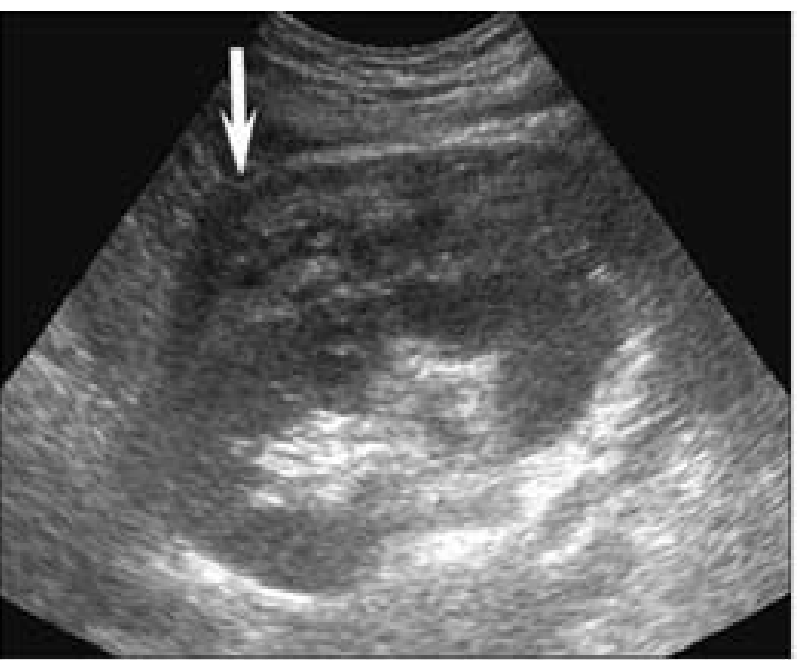

Figure 2. Perigraft Haematoma shown by arrow on ultrasound.

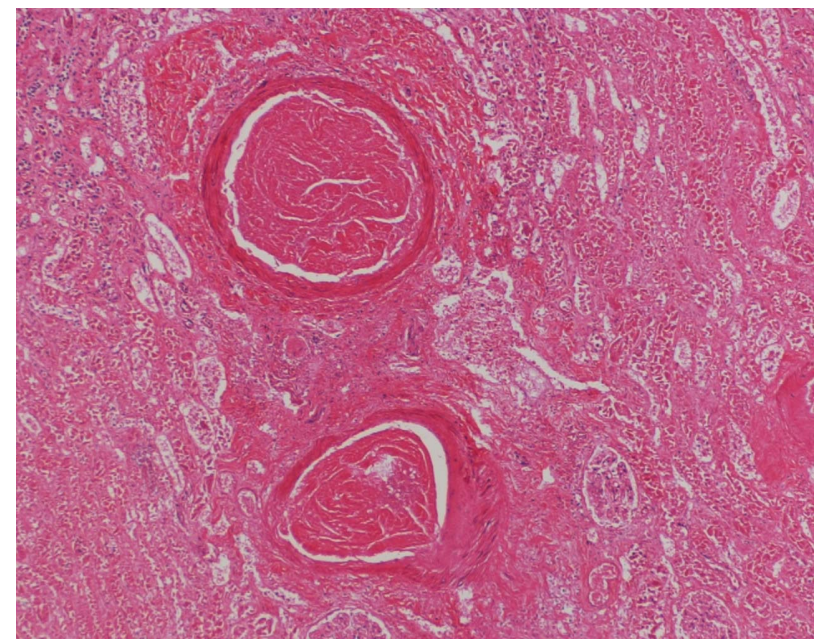

Figure 3. Slide showing the blood vessels with thrombi. (magnification $\times 400)$.

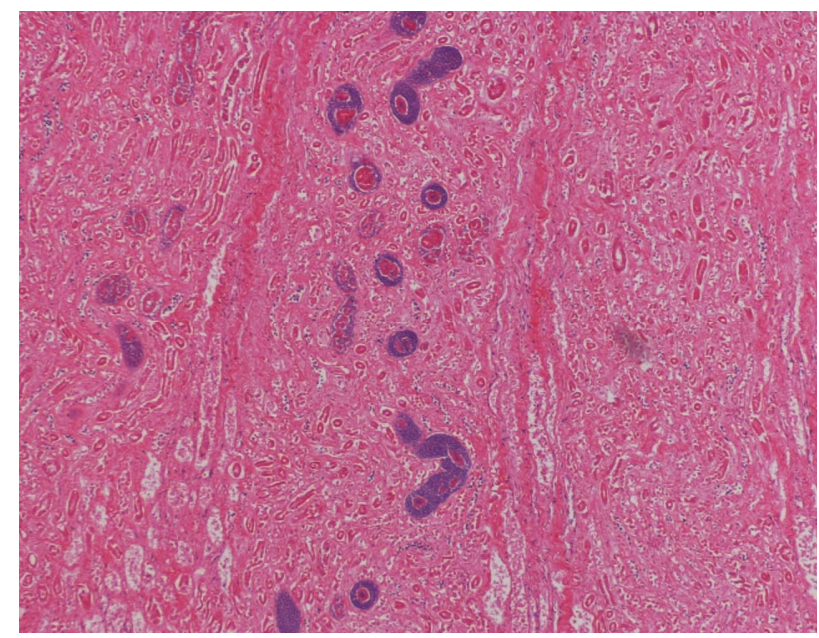

Figure 4. Tissue from the infracted kidney showing tubules with bacterial colonies (magnification $\times \mathbf{4 0 0}$ ). 


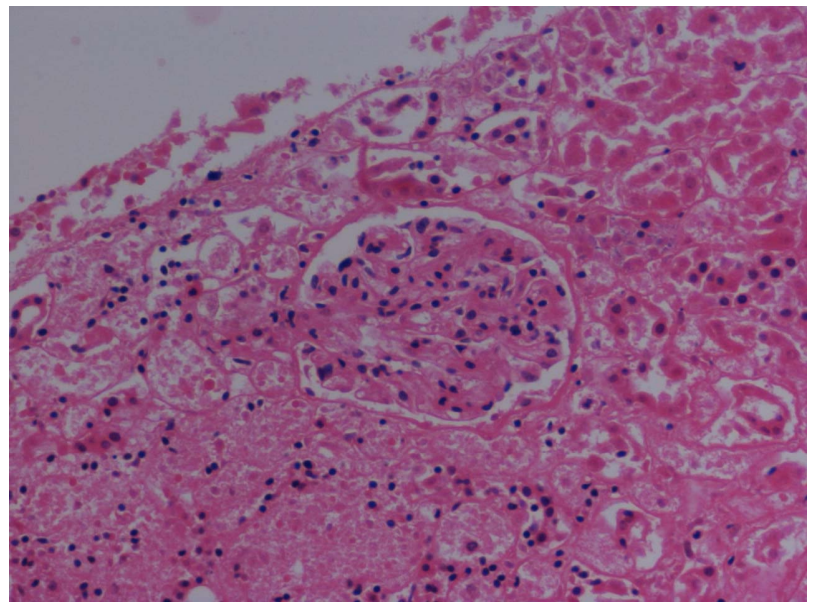

Figure 5. Infarcted tubules and glomeruli. Some of the tubules show bacterial colonies (blue-purple areas) magnification $\times 600$.

kidney with large collection of slough and thrombosis of the vascular pedicles. Graft Nephrectomy was performed. Histopathology of the specimen (Figures 3-5) showed thrombus in the blood vessels with infracted tissue containing few viable glomeruli and tubules in the periphery, blood vessels showing wide spread thrombi with necrosis of the vessel wall and interstitium showing haemorhage. The tubules were showing bacterial colonies of gram negative bacilli. There were no features of acute rejection. Patient recovered from surgery and subsequently he was discharged home on maintenance dialysis.

\section{Discussion}

Renal graft thrombosis is not a rare, complication of renal transplantation. It is a dreaded and catastrophic complication. Graft thrombosis within a month of transplantation occurs in $0.9 \%$ of transplants and account for $17 \%$ of early (within 30days of transplantation) graft failure [1].

Most common causes of renal artery thrombosis are antibody mediated rejection, hypercoagulable syndrome, technical problems associated with graft harvesting and perfusion. This is reported to be associated with small sized graft, misalignment, torsion or kinking of the renal artery. In vitro data also suggest that immunosuppressive drugs like cyclosporine and OKT3 may increase the risk of thrombosis [2]. In our patient we have no information on the use of OKT3.

Early renal vein thrombosis often results from poor surgical technique, perigraft fluid collection, compression of the common iliac veins and hypovolemia [3]. The reported incidence varies between $0.9 \%$ and $4.5 \%$ [4]. On the other hand late renal vein thrombosis have been reported in association with recurrent or denovo membranous nephropathy, ilio-femoral vein thrombosis and thrombophilic disorders [5,6].

Epidemiology data points towards increased risk of thrombosis in retransplant, those on peritoneal dialysis prior to transplantation compared to those on haemodialysis and those with atherosclerotic lesions $[3,7,8]$.

Coming to the donor related risk factors for increased risk of thrombosis Amezquita et al. have proposed that probably the right kidney from the donor as opposed to the left is the risk factor for early graft vascular thrombosis. On this basis, they further suggested for prophylactic anticoagulation or platelets antiaggregation therapy for right donor kidney implantation [9].

However, Bakir and colleagues [8], in a larger series, from a single centre, comprising of more than 550 cadaveric renal transplant recipients, have shown no association between primary renal graft thrombosis and reciepients age, sex, number of transplants, type of dialysis, pretransplant treatment with erythropoietin, antiplatelet agents, oral anticoagulation, donors age, sex, number of graft vessels, warm and cold ischemia time, site of transplantation and even the type of immunosuppressive agents used for induction like cyclosporine A or OKT3.

In our case both the renal vein and artery were thrombosed on macroscopic examination during the nephrectomy along with extensive thrombi in the intrarenal blood vessels. The graft had undergone necrosis with presence of emphysematous pyelonephritis of the graft kidney. There was no evidence of deep vein thrombosis of the lower limbs by Doppler ultrasound.

The presence of thrombus in both the arteries and veins including the small intra-renal vessels as in our case would partially exclude the role of local factors contributing to thrombosis. The predominant factor operating here being infection and its consequences like Disseminated intravascular coagulation (DIC). This could be well supported by thrombocytopenia, elevated FDP on presentation (Table 1) and the finding of gram negative bacterial colonies in the graft kidney.

The incidence of hemorrhage is much increased in the patients who are on warfarin and develop DIC. Our patient was on warfarin due to cardiac reasons and developed emphysematous pyelonephritis. The events culminated in hemorrhage followed by extensive thrombosis in the renal allograft. This was seen in the graft biopsy specimen.

Early detection, of thrombosis of the ilio-femoral vein, graft renal artery and vein, with timely surgical intervention or thrombolysis with streptokinase helps in improving the long term graft outcome $[3,10]$. However attempts at salvaging the graft by thrombolyis or by using heparin carries the risk of haemorrhage [3].

\section{Conclusion}

Thrombosis of the renal graft can be attributed to various 
Table 1. Showing the levels of $\mathrm{Hb}$, WBC, platelets, Prothrombin time PT, Firinogen degradation products and serum creatinine over time.

\begin{tabular}{|c|c|c|c|c|c|c|}
\hline Date & $\mathrm{Hb}$ gm/dl & WBC $\times 10 \times 9 /$ lit & Platelets $\times 10 \times 9 /$ lit & Prothrombin Time in seconds & $\begin{array}{c}\text { Fibrinogen degradation } \\
\text { products in mg/lit }\end{array}$ & $\begin{array}{l}\text { Serum Creatinine } \\
\text { in } \mathrm{mmol} / \mathrm{l}\end{array}$ \\
\hline $15 \mathrm{Oct}$ & 9.6 & 10.6 & 337 & 10.5 & 2.29 & $288 \mathrm{mmol} / \mathrm{l}$ \\
\hline 31 Oct & 9.5 & 22.9 & 41 & 15.8 & 7.98 & $857 \mathrm{mmol} / \mathrm{l}$ \\
\hline $02 \mathrm{Nov}$ & 10.4 & 5.3 & 95 & 11.8 & 3.99 & $599 \mathrm{mmol} / \mathrm{l}$ \\
\hline 23 Nov & 10.2 & 8.9 & 245 & 10.5 & 3.35 & $219 \mathrm{mmol} / \mathrm{l}$ \\
\hline
\end{tabular}

reasons. Once thrombosed, the loss of graft is inevitable. However, prompt diagnosis and immediate attempt to thrombolyse with streptokinase or surgical intervention with endoluminal extraction of the thrombus do bear a great promise in the immediate and long term survival of the renal allograft.

\section{REFERENCES}

[1] J. A Bertolatus, "Etiology of Renal Artery Thrombosis in Renal Transplantation,” Medscape Transplantation, Vol. 1, No. 1, 2000.

[2] E. Carlsen, "Enhancement of Procoagulant Activity in Stimulated Mononuclear Blood Cells and Monocytes by Cyclosporine,” Transplantation, Vol. 43, No. 4, 1987, pp. 543-548. doi:10.1097/00007890-198704000-00018

[3] S. Gang and M. Rajapurkar, "Vascular Complications Following Renal Transplantation” JNRT, Vol. 2, No. 1, 2009, pp. 122-132.

[4] P. Giustacchini, F. Pisanti, F. Citterio, A. M. De Gaetano, M. Castagneto and G. Nanni, "Renal Vein Thrombosis after Renal Transplantation: An Important Cause of Graft Loss,” Transplantation Proceedings, Vol. 34, No. 6, 2002, pp. 2126-2127. doi:10.1016/S0041-1345(02)02876-2

[5] M. R. First, N. Mendoza, R. K. Maryniak and M. A. Weiss, "Membranous Glomerulopathy Following Kidney Transplantation. Association with Renal Vein Thrombosis in Two of Nine Cases,” Transplantation, Vol. 38, No.
6, 1984, pp. 603-607. doi:10.1097/00007890-198412000-00011

[6] P. J. Ramirez, R. Y. Gohh, A. Kestin, A. P. Monaco and P. E. Morrissey, "Renal Allograft Loss Due to Proximal Extension of the Ileofemoral Deep Venous Thrombosis," Clinical Transplantation, Vol. 16, No. 4, 2002, pp. 310313. doi:10.1034/j.1399-0012.2002.02006.x

[7] A. Sanni, C. H. Wilson, H. Wyrley-Birch, D. Vijayanand, A. Navarro, S. Sohrabi, B. Jaques, D. Rix, N. Soomro, D. Manas and D. Talbot, "Donor Risk Factor for Renal Graft Thrombosis,” Transplantation Proceedings, Vol. 39, No. 1, 2007, pp. 138-139.

[8] N. Bakir, W. J. Sluiter, R. J. Ploeg, W. J. van Son and A. M. Tegzess, "Primary Renal Graft Thrombosis," Nephrology Dialysis Transplantation, Vol. 11, No. 1, 1996, pp. 140-147. doi:10.1093/oxfordjournals.ndt.a027030

[9] Y. Amezquita, C. Mendez, A. Fernandez, S. Caldes, J. Pascual, A. Muriel, F. J. Burgos, R. Marcen and J. Ortuno, "Risk Factors for Early Graft Thrombosis: A Case-Controlled Study in Grafts from the Same Donor," Transplantation Proceedings, Vol. 40, No. 9, 2008, pp. 28912893. doi:10.1016/j.transproceed.2008.09.014

[10] E. Renoult, L. Cormier, M. Claudon, T. Cao-Huu, L. Frimat, O. Gaucher, J. Hubert and M. Kessler, "Successful Surgical Thrombectomy of Renal Allograft Vein Thrombosis in the Early Postoperative Period,” American Journal of Kidney Diseases, Vol. 35, No. 5, 2000, p. E21. doi:10.1016/S0272-6386(00)70286-1 